\title{
EDITORIAL
}

\section{DEL ENFOQUE POBLACIONAL AL INDIVIDUAL EN LA PREVENCIÓN PRIMARIA DE LA ENFERMEDAD VASCULAR: QUE EL BOSQUE NOS DEJE VER LOS ÁRBOLES}

\author{
Rafel Ramos y Pascual Solanas \\ Unitat de Suport a la recerca de Girona. \\ Unitat docent de MFiC de Girona. \\ Institut de Investigació en Atenció Primària Jordi Gol. Institut Català de la Salut.
}

El informe de 1982 del Comité de expertos de la OMS sobre Prevención de la Enfermedad Coronaria ${ }^{1}$ ya consideró que las estrategias de prevención debían incluir tres componentes: 1) una estrategia poblacional, para intentar modificar aquellos estilos de vida, factores ambientales sociales y económicos que favorecen la presentación de la enfermedad; 2) una estrategia de prevención secundaria, para evitar recurrencias, complicaciones y evolución desfavorable de la enfermedad y 3) una estrategia para actuar en la población sin enfermedad establecida pero expuesta a un riesgo elevado con el objetivo de reducir los niveles de sus factores de riesgo. En este tercer punto son varias las circunstancias que condicionan la necesidad de establecer este orden de prioridades dentro de la prevención cardiovascular en atención primaria: la prevalencia elevada de los factores de riesgo, la elevada demanda asistencial que generan en un sistema nacional de salud universal y gratuito como es el

Correspondencia:

Rafel Ramos

IDIAP Jordi Gol

Maluquer Salvador 11

17002, Girona

Corro electrónico:

HYPERLINK "mailto:p416urrb@pgirona.scs.es"

p416urrb@pgirona.scs.es español y los discretos resultados de las intervenciones realizadas por el personal sanitario cuando se valora su efecto sobre el total de la población y en pacientes de bajo riesgo $\mathrm{o}^{2-7}$.

En este sentido, la detección de los factores de riesgo y la estimación del riesgo global del individuo son las estrategias fundamentales para priorizar acciones en prevención primaria y la principal herramienta es la ecuación de riesgo.

En este número de la Revista se incluye un artículo sobre la capacidad predictiva, comparación y consecuencias clínicas de las tablas de Framingham-Wilson y REGI$\mathrm{COR}^{8}$. Las ecuaciones de riesgo son instrumentos de predicción útiles en el cribado de enfermedades y por tanto no destinadas al diagnóstico. Se diseñaron para ser usadas como ayuda orientadora a los médicos responsables de la prevención primaria de las enfermedades cardiovasculares calculando el riesgo en pacientes de 35 a 74 años a partir de su sexo, edad, presión arterial sistólica, colesterol total, colesterol de HDL, consumo de tabaco y diagnóstico de diabetes.

Es importante partir del punto de que la ecuación adaptada de REGICOR ${ }^{9,10}$ es una adaptación de la ecuación de Wilson ${ }^{11}$ y no 
de la función publicada por Anderson et al ${ }^{12}$ como se cita en el artículo ${ }^{8}$. De la metodología de la adaptación se deriva que la comparación que se realiza en el manuscrito sea, fundamentalmente, la comparación entre dos puntos de corte. El punto de corte del $20 \%$ estimado por la ecuación de Wilson es muy cercano a un $7 \%$ estimado por REGICOR (que se aproxima al riesgo real de acontecimientos de la población). Así pues, los resultados que se obtendrían en una comparación de la aplicación de REGICOR utilizando el $7 \%$ y el $10 \%$ como puntos de corte se aproximarían a los resultados presentados en este estudio. El área bajo la curva ROC con la ecuación adaptada debería ser igual a la original. Esto se debe a que la adaptada no es más que un reescalado de la función de Wilson: un individuo que tenga un riesgo mayor que otro cuando este riesgo se mide con la ecuación adaptada, también tendrá un riesgo mayor que el otro cuando el riesgo se mida con la ecuación original. Por poner un ejemplo sencillo, es como si un niño (con las manos pequeñas) y un adulto (con las manos grandes) tuvieran que ordenar de mayor a menor diez mesas midiéndolas "a palmos". Los dos las ordenarían de la misma forma, aunque una misma mesa midiera muchos más palmos de la mano del niño que de la del adulto.

Por otro lado, aunque la interpretación de la concordancia en función de los valores del estadístico Kappa presenta cierta arbitrariedad, un valor situado entre 0,61 y 0,80 , como resulta en el estudio que se publica, se pueden interpretar como de una buena concordancia ${ }^{13}$.

Por tanto, las diferencias que se observan en la comparación realizada en el artículo se deben, fundamentalmente, al diferente punto de corte que representan y no a las diferencias en las características matemáticas entre las dos ecuaciones.

Los autores comentan las características particulares de la muestra analizada. A pesar de no presentar datos sobre prevalencia de diabetes, la media de glucemia (que se puede estimar con la tabla $3^{8}$ de 115,4 $\mathrm{mg} / \mathrm{dl}$ nos informa que la proporción de pacientes con diabetes de la muestra no es despreciable. Se trata de una población muy seleccionada, con numerosos factores de riesgo ( $78 \%$ de hipertensos, $25 \%$ de fumadores y niveles medios de colesterol total de $242,9 \mathrm{mg} / \mathrm{dl}$ ) y, por tanto, representativa de un pequeño segmento de la población (aproximadamente el 10\%) y de un nivel de riesgo elevado. Es posible, como se apunta en el artículo, que ésta sea una de las causas de las diferencias en los valores de sensibilidad y especificidad observados por Cañón et al. (28,7\% y $83,1 \%$, respectivamente) en relación a los presentados en una reciente publicación del estudio VERIFICA $(36,8 \%$ y $88,3 \%$, respectivamente) ${ }^{14}$. Así pues, los resultados de este estudio no valoran el rendimiento de la ecuación en la población general (a la que se destina su uso) en la que los grupos de bajo riesgo están mayoritariamente representados.

En realidad, las ecuaciones de riesgo estiman el exceso de riesgo que tiene un individuo de presentar un acontecimiento en un periodo de tiempo en relación al riesgo promedio de la población en la que vive. Son herramientas diseñadas para estimar el riesgo en una población con una distribución común en relación a factores de riesgo, sexo y edad, y ello conlleva que su aplicación a escala individual implique alguna limitación.

Una de las características de las funciones de riesgo es una sensibilidad que se sitúa alrededor del $40 \%$ en función de las diferentes ecuaciones y puntos de corte definidos ${ }^{14}$. Cuanto más compleja es la etiopatogenia de una enfermedad y más factores intervienen, más difícil es expresar en un modelo matemático toda su variabilidad. La enfermedad coronaria es multifactorial y se desarrolla en función de la presencia, combinación e interacción de numerosos 
factores genéticos y ambientales, algunos de ellos desconocidos, y ello comporta limitaciones para construir un modelo matemático que nos explique toda su variabilidad y que tenga una gran precisión. Evidentemente, este hecho repercute en la sensibilidad de todos los modelos matemáticos en general a la hora de predecir un acontecimiento (si los comparamos al mismo punto de corte). En países de alta incidencia la sensibilidad de la función original de Framingham se sitúa alrededor del $40 \%{ }^{15}$. Si bien esta sensibilidad no sería admisible para una prueba diagnóstica, la importancia de esta característica es mucho menor para una prueba de cribado de riesgo de enfermedad. En nuestro contexto poblacional resulta inevitable que al ser muy elevado el número de personas con riesgo cardiovascular bajo, una importante proporción de casos se producirá en este grupo poblacional, meramente por la mayor cantidad de personas que contiene.

Cabe recordar que el cribado poblacional de riesgo vascular intenta estimar la probabilidad de presentar una enfermedad en el futuro, por tanto aún no presente. Este hecho contrasta con otros cribados poblacionales como el de neoplasia de mama en el que se intenta diagnosticar la enfermedad en estadios precoces; aún así la sensibilidad de la mamografía en el cribado poblacional varía entre el 39 y el $86 \%$ en función de la calidad de la exploración y del grupo de población estudiado ${ }^{16}$.

Es fundamental ser conscientes de que además de las características propias de la ecuación, la sensibilidad va a depender esencialmente del punto de corte elegido, de manera que disminuyendo el dintel de riesgo aumentamos la sensibilidad pero siempre a costa de empeorar la especificidad. Alcanzar el 100\% de sensibilidad para detectar a los individuos que presentarán un evento coronario a 10 años implicaría tratar a toda la población, y ni aun así se conseguiría evitar todos los acontecimientos, ya que la eficacia de los métodos preventivos es limitada. El nivel de esfuerzo que se realizará en la prevención será determinado, en gran medida, por el punto de corte en el riesgo cardiovascular a partir del que se aconseje actuar farmacológicamente. Dicho punto de corte es indiscutiblemente arbitrario aunque en su elección debe valorarse, principalmente, la especificidad de la prueba ya que el impacto de la decisión tiene mayor influencia en el número de tratados innecesariamente (falsos positivos) que sobre el número de tratados correctamente (verdaderos positivos). También será importante tener en cuenta la evidencia del beneficio del tratamiento farmacológico sobre la morbi-mortalidad de dicha población. Se desconoce la eficacia y eficiencia del tratamiento hipolipemiante en grupos de población de bajo riesgo. El tercer informe del National Cholesterol Education Program $^{2}$ afirma que en individuos con un riesgo inferior al $10 \%$ a 10 años el tratamiento hipolipemiante no es coste-efectivo y que esta relación de coste-efectividad decrece al disminuir el riesgo de la población. Además, no se han publicado ensayos clínicos que aporten pruebas suficientes sobre el beneficio del tratamiento hipolipemiente en poblaciones con un riesgo medio inferior al $12 \%{ }^{17-19}$.

En la actualidad, no disponemos de un sistema alternativo a las ecuaciones de riesgo para realizar la primera aproximación en la clasificación y ordenación de la prevención primaria, ya que la opinión subjetiva de los profesionales sobre el riesgo de los pacientes es claramente menos precisa que la estimación que nos proporcionan las tablas de riesgo ${ }^{20,21}$.

El resultado de un primer cribado con la función de riesgo debe llevar a la consideración de otros factores complementarios como los antecedentes familiares de enfermedad cardiovascular precoz, la presencia de arteriopatía periférica asintomática (índice tobillo/brazo $<0,9$ ), la hipertrigli- 
ceridemia, insuficiencia renal o microalbuminuria, la diabetes de larga evolución, la obesidad o perímetro de cintura elevado, o incluso la calidad de la alimentación, los hábitos de actividad física o, como se ha publicado recientemente, la condición social ${ }^{22,23}$. Algunas de las condiciones mencionadas pueden excluir directamente a los pacientes del cálculo de riesgo y situarlo en riesgo elevado sin más consideraciones. En los pacientes de riesgo bajo o medio, la valoración clínica puede inducir a reclasificar como con riesgo alto a los individuos que presenten un perfil desfavorable por la presencia de algunos de los factores descritos. Además, en un futuro tal vez no muy lejano, se podrá disponer de pruebas de imagen no invasivas adecuadas en la valoración de determinados casos.

En la población con características similares a la del artículo de Cañón et al., en la que existe una proporción importante de pacientes con riesgos próximos al límite del $10 \%$ (recordemos que la media de riesgo calculado con REGICOR en hombres fue de $8,5 \%$ ) es donde mayor utilidad tiene la valoración de otros factores de riesgo ausentes en las ecuaciones para clasificar correctamente a los pacientes.

No obstante, la prevención con consejo sobre la mejora de los estilos de vida, apoyada por evidencia científica sólida, puede aplicarse sin restricciones, y debe indicarse de manera sistemática e independientemente del nivel de riesgo cardiovascular. La etiología multifactorial de las enfermedades vasculares obliga a pensar que las estrategias preventivas deben ser intervenciones también multifactoriales.

De todas estas consideraciones se derivan dos conclusiones fundamentales:

La primera es que los parámetros de sensibilidad de las ecuaciones predictivas no deben desorientar al lector sobre su enorme utilidad, siempre y cuando se entienda como una herramienta poblacional útil en la primera aproximación individual al cribado de riesgo y toma de decisiones. Y la segunda es que las estrategias poblacionales de prevención pretenden y deben establecer un marco de directrices en aspectos básicos de la prevención vascular, no obstante, el criterio médico debe ser la base fundamental para la toma de decisiones finales a nivel individual. La valoración de las medidas adecuadas para la aplicación a cada individuo debe tener en cuenta el contexto biopsicosocial y las particularidades de cada caso.

\section{AGRADECIMIENTOS}

Los autores agradecen los valiosos comentarios de Joan Sala, Jaume Marrugat y Joan Vila.

\section{BIBLIOGRAFÍA}

1. Prevention of coronary heart disease. Report of a WHO Expert Committee. WHO Technical Report Series 678. Geneva: World Health Organization; 1982 .

2. Third Report of the National Cholesterol Education Program (NCEP). Expert Panel on Detection, Evaluation, and Treatment of High Blood Cholesterol in Adults (Adult Treatment panel III) final report. Circulation. 2002; 106: 3143-21.

3. Kannel WB, D'Agostino RB, Sullivan L, Wilson PW. Concept and usefulness of cardiovascular risk profiles. Am Heart J. 2004; 148: 16-26.

4. Ebrahim S, Smith GD. Systematic review of randomised controlled trials of multiple risk factor interventions for preventing coronary heart disease. BMJ. 1997; 314: 1.666-674.

5. Wonderling D, Langham S, Buxton M, Normand $\mathrm{C}, \mathrm{McDermott} \mathrm{C}$. What can be concluded from the Oxcheck and British Family Heart Studies. Commentary on cost effectiveness analyses. BMJ. 1996; 312: 1.274-78.

6. Banegas JR, Rodríguez Artalejo F, Cruz JJ, Guallar $\mathrm{P}$, Rey J. Blood pressure in Spain: distribution, awa- 
reness, control, and benefits of a reduction in average pressure. Hypertension. 1998; 32: 998-1.002.

7. Sierra A, Ruilope LM, Coca A, Luque-Otero M, (Grupo Español de Estudios Cardiovasculares). Relación entre el perfil de riesgo cardiovascular y la selección y utilización de los fármacos antihipertensivos. Med Clin (Barc). 2000; 115:41-45.

8. Cañón Barroso L, Díaz Herrera N, Cruces Muro E, Nieto Hernández T, Garrote Florencio T y Buitrago F. Capacidad predictiva, comparación y consecuencias clínicas de las tablas de framingham-wilson y REGICOR. Rev Esp Salud Publica. 2007.; 81:35364.

9. Marrugat J, D'Agostino R, Sullivan L et al. An adaptation of the Framingham coronary heart disease risk function to European Mediterranean areas. J Epidemiol Community Health. 2003; 57:634-8.

10. D'Agostino RB, Grundy S, Sullivan LM, Wilson P. CHD Risk Prediction Group.Validation of the Framingham Coronary Heart Disease Prediction Scores: Results of a Multiple Ethnic Groups Investigation. JAMA. 2001; 286:180-7.

11. Wilson PWF, D'Agostino RB, Levy D, Belanger AM, Silbershatz H, Kannel WB. Prediction of coronary heart disease using risk factor categories. Circulation. 1998; 97:1837-47.

12. Anderson KM, Wilson PWF, Odell PM, Kannel WB. An updated coronary risk profile: A statement for health professionals. Circulation. 1991; 83:356-62.

13. Altman DG. Practical statistics for medical research. New York: Chapman and Hall; 1991.

14. Comín E, Solanas P, Cabezas C, Subirana I, Ramos R, Gené-Badía J, Cordón F, Grau M, Cabré-Vila JJ y Marrugat J. Rendimiento de la estimación del riesgo cardiovascular en España mediante la utilización de distintas funciones. Rev Esp Cardiol 2007; 60:693-702.
15. Jones AF, Walker J, Jewkes C, Game FL, Bartlett WA, Marshall T, Bayly GR. Comparative accuracy of cardiovascular risk prediction methods in primary care patients. Heart. 2001; 85:37-43.

16. Breast cancer screening: a summary of the evidence for the U.S. Preventive Services Task Force. Ann Intern Med. 2002; 137:347-60.

17. Ramos R, Marrugat J. Valoración del riesgo cardiovascular en la población. En: Alfonso del Río Ligorit, editor. Manual de cardiología preventiva. Madrid: SMC; 2005; p. 43-45.

18. Miguel F, García A, Montero MJ. Prevención primaria con estatinas, consensos y tablas de riesgo. Aten Primaria. 2005; 36:31-8.

19. The Atorvastatin Study for Prevention of Coronary Heart Disease Endpoints in Non-InsulinDependent Diabetes Mellitus (ASPEN) Robert H. Knopp, MD1, Michael d'Emden, MD2, Johan G. Smilde, MD, PHD3, Stuart J. Pocock, PHD4 on behalf of the ASPEN Study Group. Diabetes Care 2006; 29:1478-85.

20. Montgomery AA, Fahey T, MacKintosh C, Sharp DJ, Peters TJ. Estimation of cardiovascular risk in hypertensive patients in primary care. Br J Gen Pract. 2000; 50:127-8.

21. Hanon O, Franconi G, Mourad JJ, Baleydier A, Croce I, Girerd X. The estimation of cardiovascular risk in hypertensive patients is not modified by management of the hypertension. Arch Mal Coeur Vaiss. 2000; 93:943-7.

22. Hippisley-Cox J, Coupland C, Vinogradova Y, Robson J, May M, Brindle P. Derivation and validation of QRISK, a new cardiovascular disease risk score for the United Kingdom: prospective open cohort study. BMJ. 2007; 335:136.

23. Bonneux L. Cardiovascular risk models. BMJ. 2007; 335:107-8. 